Delft University of Technology

\title{
The spatially resolved [C II] Cooling line deficit in galaxies
}

Smith, J. D T; Croxall, Kevin; Draine, Bruce; Looze, Ilse De; Sandstrom, Karin; Armus, Lee; Beirão, Pedro; Bolatto, Alberto; Boquien, Mederic; Brandl, Bernhard

DOI

10.3847/1538-4357/834/1/5

Publication date

2017

Document Version

Final published version

Published in

The Astrophysical Journal: an international review of astronomy and astronomical physics

\section{Citation (APA)}

Smith, J. D. T., Croxall, K., Draine, B., Looze, I. D., Sandstrom, K., Armus, L., Beirão, P., Bolatto, A., Boquien, M., Brandl, B., Crocker, A., Dale, D. A., Galametz, M., Groves, B., Helou, G., Herrera-Camus, R., Hunt, L., Kennicutt, R., Walter, F., \& Wolfire, M. (2017). The spatially resolved [C II] Cooling line deficit in galaxies. The Astrophysical Journal: an international review of astronomy and astronomical physics, 834(1), [5]. https://doi.org/10.3847/1538-4357/834/1/5

Important note

To cite this publication, please use the final published version (if applicable).

Please check the document version above.

\section{Copyright}

Other than for strictly personal use, it is not permitted to download, forward or distribute the text or part of it, without the consent of the author(s) and/or copyright holder(s), unless the work is under an open content license such as Creative Commons.

\section{Takedown policy}

Please contact us and provide details if you believe this document breaches copyrights.

We will remove access to the work immediately and investigate your claim. 


\title{
THE SPATIALLY RESOLVED [C II] COOLING LINE DEFICIT IN GALAXIES
}

\author{
J. D. T. Smith ${ }^{1,2}$, Kevin Croxall ${ }^{3,4}$, Bruce Draine ${ }^{5}$, Ilse De Looze ${ }^{6}$, Karin Sandstrom ${ }^{7}$, Lee Armus ${ }^{8}$, Pedro Beirão 9 , \\ Alberto Bolatto $^{10}$, Mederic Boquien ${ }^{11}$, Bernhard Brandl ${ }^{12,13}$, Alison Crocker ${ }^{14}$, Daniel A. Dale ${ }^{15}$, \\ Maud Galametz ${ }^{16}$, Brent Groves ${ }^{17}$, George Helou ${ }^{18}$, Rodrigo Herrera-Camus ${ }^{19}$, Leslie Hunt ${ }^{20}$, Robert Kennicutt ${ }^{6}$, \\ FABIAN WALTER $^{2}$, AND MARK WOLFIRE ${ }^{10}$ \\ ${ }^{1}$ Ritter Astrophysical Research Center, University of Toledo, Toledo, OH 43606, USA; jd.smith@utoledo.edu \\ ${ }^{2}$ Max-Planck-Institut für Astronomie, Königstuhl 17, D-69117 Heidelberg, Germany \\ ${ }^{3}$ Department of Astronomy, The Ohio State University, $140 \mathrm{~W} 18$ th Avenue, Columbus, OH 43210, USA \\ ${ }^{4}$ Illumination Works LLC, 5650 Blazer Parkway, Suite 152, Dublin, OH 43017, USA \\ ${ }^{5}$ Department of Astrophysical Sciences, Princeton University, Princeton, NJ 08544, USA \\ ${ }^{6}$ Institute of Astronomy, University of Cambridge, Madingley Road, Cambridge, CB3 OHA, UK \\ ${ }^{7}$ Center for Astrophysics and Space Sciences, University of California, San Diego, 9500 Gilman Drive, La Jolla, CA 92093, USA \\ ${ }^{8}$ Spitzer Science Center, California Institute of Technology, MC 314-6, Pasadena, CA 91125, USA \\ ${ }^{9}$ Observatoire de Paris, 61 avenue de l'Observatoire, F-75014 Paris, France \\ ${ }^{11}$ Department of Astronomy, University of Maryland, College Park, MD 20742, USA
${ }^{11}$ Unidad de Astronoma, Fac. Cs. Bsicas, Universidad de Antofagasta, Avda. U. de Antofagasta 02800, Antofagasta, Chile \\ ${ }^{12}$ Leiden Observatory, Leiden University, P.O. Box 9513, 2300 RA Leiden, The Netherlands \\ ${ }^{13}$ Faculty of Aerospace Engineering, Delft University of Technology, Kluyverweg 1, 2629 HS Delft, The Netherlands \\ ${ }^{14}$ Department of Physics, Reed College, Portland, OR 97202, USA \\ ${ }^{15}$ Department of Physics and Astronomy, University of Wyoming, Laramie, WY 82071, USA \\ ${ }^{16}$ European Southern Observatory, Karl-Schwarzschild-Str. 2, 85748 Garching-bei-München, Germany \\ ${ }^{17}$ Research School of Astronomy \& Astrophysics, Australian National University, Canberra, ACT 2611, Australia \\ ${ }^{18}$ Infrared Processing and Analysis Center, California Institute of Technology, 1200 E. California Boulevard, Pasadena, CA 91125, USA \\ ${ }_{19}$ Max Planck Institute for Extraterrestrial Physics Garching, Germany \\ ${ }^{20}$ INAF-Osservatorio Astrofisico di Arcetri, Largo E. Fermi 5, I-50125 Firenze, Italy \\ Received 2016 July 1; revised 2016 November 2; accepted 2016 November 3; published 2016 December 22
}

\begin{abstract}
We present [C II] $158 \mu \mathrm{m}$ measurements from over 15,000 resolved regions within 54 nearby galaxies of the KINGFISH program to investigate the so-called [C II] "line-cooling deficit" long known to occur in galaxies with different luminosities. The [C II]/TIR ratio ranges from above $1 \%$ to below $0.1 \%$ in the sample, with a mean value of $0.48 \pm 0.21 \%$. We find that the surface density of $24 \mu \mathrm{m}$ emission dominates this trend, with [C II]/TIR dropping as $\nu I_{\nu}(24 \mu \mathrm{m})$ increases. Deviations from this overall decline are correlated with changes in the gas-phase metal abundance, with higher metallicity associated with deeper deficits at a fixed surface brightness. We supplement the local sample with resolved [C II] measurements from nearby luminous infrared galaxies and highredshift sources from $z=1.8-6.4$, and find that star formation rate density drives a continuous trend of deepening [C II] deficit across six orders of magnitude in $\Sigma_{\mathrm{SFR}}$. The tightness of this correlation suggests that an approximate $\Sigma_{\text {SFR }}$ can be estimated directly from global measurements of [C II]/TIR, and a relation is provided to do so. Several low-luminosity active galactic nucleus (AGN) hosts in the sample show additional and significant central suppression of $[\mathrm{CII}] / \mathrm{TIR}$, but these deficit enhancements occur not in those AGNs with the highest X-ray luminosities, but instead those with the highest central starlight intensities. Taken together, these results demonstrate that the [C II] line-cooling line deficit in galaxies likely arises from local physical phenomena in interstellar gas.
\end{abstract}

Key words: dust, extinction - galaxies: ISM - infrared: galaxies - techniques: spectroscopic

\section{INTRODUCTION}

The flow of radiative energy through the gas and dust comprising the interstellar medium (ISM) of galaxies strongly impacts their observational and physical properties. Indeed, the process of star formation itself is controlled in part by the balance between the radiative heating and cooling in starforming clouds of gas and dust (e.g., Krumholz et al. 2011). With high ultraviolet (UV) opacity, dust grains absorb and reradiate a significant fraction of a galaxy's radiative energy (from a few percent up to nearly 100 percent in the most infrared-luminous objects, Sanders \& Mirabel 1996). The smallest of these dust grains have small heat capacities, and so are stochastically heated to high non-equilibrium temperatures (Draine \& Li 2001). With their modest ionization energies ( $\sim 6-9 \mathrm{eV}$, Witt et al. 2006) and large cross-sections, these small grains, including polycyclic aromatic hydrocarbons
(PAHs), are also the dominant source of the photoelectrons that energetically couple the radiation field to the gas outside of hydrogen-ionized zones (Bakes \& Tielens 1994).

Neutral gas cools predominantly through collisional excitation of abundant ions with low-lying excited states. With a low first ionization potential $(11.26 \mathrm{eV})$ well below the Lyman limit, atomic carbon is found in singly ionized form in a wide range of environments. Together with its high abundance and modest first excitation energy, this makes ionized carbon a highly effective coolant of neutral atomic and even diffuse molecular gas. Indeed, for this reason, the most luminous emission line of a galaxy is typically [C II] $158 \mu \mathrm{m}$ (e.g., Stacey et al. 1991). This high line luminosity (up to several percent of the total infrared output) also makes [C II] a promising diagnostic tool for mapping the key physical properties of galaxies in the early universe, including star formation rate, 
kinematics, size, virial mass and molecular content. One of ALMA's primary science drivers involves imaging [C II] in Milky-Way-type galaxies at redshifts greater than 3, and interferometers like IRAM/PdBI and ALMA are now detecting [C II] in galaxies beyond redshift $z=6-7$ (e.g., Venemans et al. 2012; Capak et al. 2015; Maiolino et al. 2015; Willott et al. 2015).

Helou et al. (2001) used the near constancy of [C II] relative to PAH emission in support of models that stressed the importance of very small dust grains to photoelectric heating of neutral gas. But for stellar populations drawn from realistic star formation histories, the small grains that heat gas via photoelectrons, and the larger grains that re-process the bulk of absorbed UV/Optical radiative energy into the infrared, do not differ substantially in their contributions to absorption of starlight (e.g., Crocker et al. 2013). Thus, the rate of gas cooling via [C II] might be expected to closely track the total infrared (TIR, 3-1100 $\mu \mathrm{m}$ ) emission of galaxies-[C II]/TIR should remain approximately constant. Indeed the suitability of [C II] as a direct star formation tracer, which follows from this expectation, has been given considerable attention (Boselli et al. 2002; Farrah et al. 2013; De Looze et al. 2014; HerreraCamus et al. 2015; Kapala et al. 2015).

And yet, relative to the heating rate inferred from bolometric infrared luminosity, the line luminosity of this principal coolant is found to vary by orders of magnitude among different galaxy types and environments (Malhotra et al. 1997, 2001; Luhman et al. 2003; Brauher et al. 2008; Stacey et al. 2010; GraciáCarpio et al. 2011; Díaz-Santos et al. 2013). This so-called [C II] cooling line deficit problem, first revealed as an unexpectedly low [C II] luminosity among local ultra-luminosity infared galaxies (ULIRGs), has proven difficult to reconcile, with possible explanations invoking physical impacts on either the coupling of radiation to the gas via dust grains, or on the nature of the subsequent gas cooling itself.

The Herschel Space Observatory has for the first time delivered the combination of sensitivity and spatial resolution necessary to map the fundamental cooling lines on subkiloparsec scales in nearby galaxies. KINGFISH (Key Insights on Nearby Galaxies-a Far-Infrared Survey with Herschel; Kennicutt et al. 2011) has investigated a diverse sample of galaxies in the nearby universe $(d \lesssim 30 \mathrm{Mpc})$. Here we explore resolved maps of [C II] across the KINGFISH sample in order to delineate the principal physical drivers of the deficit itself. In Section 2 we detail the Herschel and supporting observations and data reduction and demonstrate the resulting quality of the [C II] maps; in Section 3 we highlight the impact of surface brightness and metallicity on the deficit; in Section 4 we evaluate the role played by star formation rate density, both locally in normal and luminous infrared galaxies, and at high redshift; in Section 5 we investigate the spatially resolved impact of active galactic nuclei (AGNs), concluding with discussions in Sections 6 and 7.

\section{OBSERVATIONS AND DATA REDUCTION}

\subsection{PACS Observations}

Herschel/PACS was used to perform far-IR mapped spectral observations of the [C II] $158 \mu \mathrm{m}$ line as part of the Herschel Open Time Key Program KINGFISH. Observations targeted radial strips centered on galactic nuclei as well as additional extranuclear regions. The targeted galaxies cover a wide range of star formation rate $\left(0.02-11 M_{\odot} \mathrm{yr}^{-1}\right)$, infrared-to-optical luminosity ratio $(0.02-6.2)$, and total gas mass $\left(10^{7.8}\right.$ $\left.10^{10.3} M_{\odot}\right)$. For more information on the target selection, including the mapped regions within sample galaxies, see Kennicutt et al. (2011).

All PACS spectral observations were obtained in the UnChopped Mapping mode and reduced using the Herschel Interactive Processing Environment (HIPE) version 11.2637. Reductions applied the standard spectral response functions and flat-field corrections, flagged instrument artifacts and bad pixels, and subtracted dark current. Transients caused by thermal instabilities were removed through custom treatment designed for the KINGFISH Pipeline. Specific information on data reduction is contained in Croxall et al. (2012) and the KINGFISH Data Products Delivery (DR3) Users Guide. ${ }^{21}$ Flux maps were obtained by fitting single Gaussian profiles to each projected pixel, with care taken to avoid spurious detection of spectral artifacts. When Gaussian fitting failed (for example due to appreciable velocity broadening within the region), a straight integration was adopted. Line fits employed iterative velocity tuning, initialized with velocities from region-matched velocity measurements of atomic hydrogen from THINGS (Walter et al. 2008), where available. Flux calibration of PACS data yield absolute flux uncertainties of $\sim 15 \%$ with relative flux uncertainties between each Herschel pointing within a galaxy of $\sim 10 \%$.

Circular extraction regions of $11^{\prime \prime}$ diameter were tiled over the available fully sampled [C II] mapping area in the 54 [C II]mapped KINGFISH galaxies. This extraction size is well matched to the resolution PACS delivers at $158 \mu \mathrm{m}$, as well as that of the convolved ancillary data sets (see below). After masking for unreliable line fits and cutting at $\mathrm{S} / \mathrm{N}=4$, approximately 15,000 distinct regions with [C II] detection remained, ranging in physical diameter from $0.17-1.6 \mathrm{kpc}$ (median $0.65 \mathrm{kpc}$ ). Of the regions, $88 \%$ have [C II] detected with $\mathrm{S} / \mathrm{N}>5,60 \%$ have $\mathrm{S} / \mathrm{N}>10$, and $33 \%$ have $\mathrm{S} /$ $\mathrm{N}>20$. One galaxy characterized by deep deficits-the nascent starburst or embedded AGN host galaxy NGC 1377 (Roussel et al. 2006; Aalto et al. 2016)—was omitted from the sample, as it has extreme attenuation conditions and an ambiguous central power source (see also Herrera-Camus et al. 2015).

\subsection{Ancillary Data}

Additional Spitzer/SINGS and KINGFISH photometric maps were used to assess the total infrared power, all convolved to match the PACS $160 \mu \mathrm{m}$ channel resolution (Aniano et al. 2012). GALEX far-ultraviolet (FUV; $0.155 \mu \mathrm{m}$ ) maps, used for accurate star formation measurements at low surface brightness, were similarly convolved. Maps of luminous infrared galaxies resolved at $24 \mu \mathrm{m}$ from GOALS (Armus et al. 2009) were convolved to match the $160 \mu \mathrm{m}$ resolution using the convolution kernel methodology of Aniano et al. (2011). To minimize any possible effects of starlight contamination at shorter wavelengths (see, e.g., Kapala et al. 2015), the calibration of total infrared (TIR, 3-1100 $\mu \mathrm{m}$ ) luminosity was performed utilizing 70,100, and $160 \mu \mathrm{m}$ photometry, which closely tracks full-SED integrated TIR luminosity (Galametz et al. 2013).

\footnotetext{
$\overline{21} \mathrm{ftp}$ ///hsa.esac.esa.int/LEGACY_PRODUCTS/UPDP/KINGFISH-DR3/ KINGFISH_DR3.pdf
} 

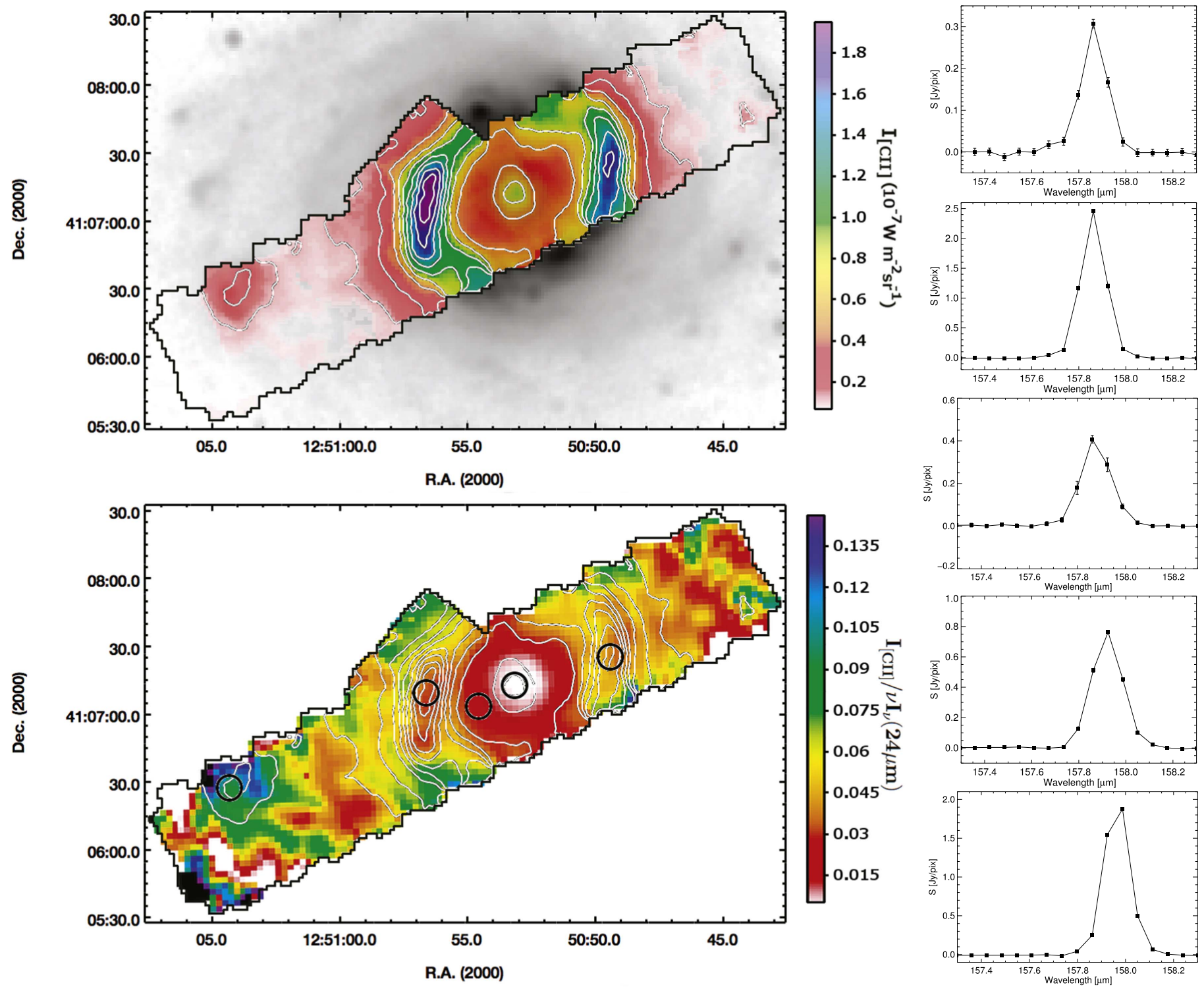

Figure 1. Example of the [C II] line maps produced from Herschel/PACS spectral mapping in KINGFISH. Above and left, the [C II] contours and intensity-matched color scale are overlaid on a Spitzer/MIPS $24 \mu \mathrm{m}$ image of KINGFISH galaxy NGC 4736. Coverage is along radial strips, supplemented by selected extranuclear targets (in this example extending the strip along the star-forming ring to the north). Below it, the $I_{[\mathrm{C} \mathrm{II}} / \nu I_{\nu}(24 \mu \mathrm{m})$ ratio map (colors, with $I_{[\mathrm{C} \text { II] }}$ contours) demonstrates the widely varying fractional [C II] intensity. Black circles indicate locations of five example [C II] extraction regions, each $11^{\prime \prime}$ in diameter. The corresponding [C II] spectra from regions left to right are arranged from top to bottom, covering a faint outer disk region, inner arm regions, the bright star-forming ring, and the nucleus.

\subsection{Resulting Line Maps}

The high sensitivity realized in Herschel/PACS unchopped mapping observations translates to significant [C II] detection in most map pixels. An example of the [C II] maps covering radial strips and selected extranuclear regions of 54 KINGFISH galaxies is given in Figure 1 for starburst-ring and low-luminosity AGN host galaxy NGC 4736 (see also van der Laan et al. 2015). Strong variation of [C II] brightness relative to the underlying $24 \mu \mathrm{m}$ emission map is seen in the $I_{[\mathrm{C} \mathrm{II}} / \nu I_{\nu}(24 \mu \mathrm{m})$ map in the lower panel. Example extracted [C II] line profiles from bright, intermediate, and faint areas of the spectral cube are included. In most cases, the lines are unresolved at the Herschel/PACS spectral resolution of $\sim 239 \mathrm{~km} \mathrm{~s}^{-1}$ at the [C II] line (see de Blok et al. 2016).
A complete compendium of KINGFISH line fluxes, spectral cubes, and related data is under preparation. Herrera-Camus et al. (2015) used the KINGFISH [C II] data set to provide updated star formation rate calibrations of the line (see Section 4). Here we investigate variations in [C II]/TIR with important intensive (i.e., local, not global) physical properties of galaxies, including surface brightness, gas-phase metallicity, star formation rate density, and AGN proximity.

\section{THE RESOLVED LINE DEFICIT IN NEARBY GALAXIES}

While early surveys uncovered an apparent dependence of the fractional [C II] line power on galaxies' bulk infrared luminosity (e.g., Malhotra et al. 2001; Luhman et al. 2003), with the highest luminosity galaxies exhibiting the deepest 
deficits, the advent of [C II] surveys at redshifts $z \gtrsim 1$ has completely altered this viewpoint. In the nearby universe, to good approximation, global luminosity serves as a reliable parameterization of the strongly varying local heating conditions in the ISM, which must underly the deficit, perhaps due to strong correlation between merger activity and high infrared luminosity. But this is emphatically not the case at earlier epochs, where wide ranges of ISM conditions exist even at the highest infrared luminosities. This is well summarized by Brisbin et al. (2015), who compile local and recent higher redshift results to demonstrate conclusively that the global infrared luminosity of a galaxy is a very poor predictor of its deficit. For example, at far-infrared (FIR, 40-122 $\mu \mathrm{m}$ ) luminosities $L_{\mathrm{FIR}}>10^{12.5} L_{\odot},[\mathrm{C} \mathrm{II}] / \mathrm{FIR}$ is not uniformly low as is found in local ULIRGS, but instead ranges over more than two orders of magnitude, and can in fact exceed the value seen in local star-forming systems more than a thousand times less luminous. This fits directly with the developing evolutionary framework in which the highest luminosity systems at earlier epochs arise not exclusively from mergers but increasingly in large-scale, cooler extended starbursts driven by the direct accretion of cold gas from the richer available reservoirs (e.g., Pope et al. 2008; MenéndezDelmestre et al. 2009; Symeonidis et al. 2013). Results from the $I S O$ satellite provided another viewpoint-that deficits are most strongly correlated with the infrared dust color temperature-an intrinsic indirect measure of the intensity of the interstellar radiation field (e.g., $I(60 \mu \mathrm{m}) / I(100 \mu \mathrm{m})$, Malhotra et al. 2001; Brauher et al. 2008). Other recent results have extended the breakdown of infrared luminosity as a key driver of the cooling deficit to redshifts as low as $z \sim 0.2$ (Ferkinhoff et al. 2014; Magdis et al. 2014; Rigopoulou et al. 2014; Brisbin et al. 2015; Ibar et al. 2015). And some have abandoned $L_{\mathrm{IR}}$ as a driving parameter in favor of intrinsic quantities such as luminosity per unit gas mass (which relates to the efficiency of star formation, Graciá-Carpio et al. 2011). This leaves open the question of which physical parameters of the star-forming ISM dominate the strong observed deficit trends. Spatially resolved samples with detailed gas and dust diagnostics on subkiloparsec scales are ideally suited to exploring this. Here we consider two alternative galaxy characteristics which together explain the bulk of the variation in [C II]/TIR found in local galaxies: infrared surface brightness (which relates to the surface density of star formation), and gas-phase metallicity, 12 $+\log (\mathrm{O} / \mathrm{H})$.

\subsection{Surface Brightness}

A number of studies have tracked the [C II] deficit on subkiloparsec scales in galaxies. Nakagawa et al. (1998) reported balloon-borne [C II] observations of a large portion of the Galactic plane, finding [C II]/FIR decreases in star-forming regions and the Galactic center. Beirão et al. (2012) found deficit variations in the starburst-ring KINGFISH galaxy NGC 1097, and Croxall et al. (2012) expanded this work to an additional galaxy, NGC 4559, uncovering changes in the ratio of [C II] to PAH emission correlated with the charge state of PAH grains. Kramer et al. (2013) and Kapala et al. (2015) found that the fractional luminosity of [C II] drops also toward the centers of M33 and M31, respectively. On larger, but still resolved scales, Díaz-Santos et al. (2014) found that the deficit in the luminous infrared galaxies (LIRGs) of the GOALS sample was mostly confined to their starbursting nuclei, and that infrared surface brightness anti-correlated with [C II]/FIR. Lutz et al. (2015) extended this to quasar host galaxies and also found a tight trend of [C II]/FIR with FIR surface brightness.

Figure 2 demonstrates the trends of [C II]/TIR in $\sim 15,000$ regions drawn from the full KINGFISH sample. Regions within $750 \mathrm{pc}$ of the centers of those galaxies optically classified as AGNs or composite sources are excluded from this plot (but see Section 5). The full sample yields $\langle[\mathrm{C}$ II $] / \mathrm{TIR}\rangle=$ $0.48 \pm 0.21 \%$ by arithmetic average, and $\langle[\mathrm{C}$ II $] / \mathrm{TIR}\rangle=$ $0.46_{-0.15}^{+0.18} \%$ for the median and inner $68 \%$ of regions. Regions are binned in logarithmic intervals of $\nu I_{\nu}(24)$ and [C II]/TIR when at least nine points fall in a bin, and are otherwise plotted individually. The density of regions falling in each bin is shown linearly scaled in the inset (418 regions maximum). The full sample median at each $\nu I_{\nu}$ (24) bin (black central solid line) demonstrates the strong and monotonic drop of the fractional cooling line luminosity with $24 \mu \mathrm{m}$ surface brightness. The trend was found to be more significant with surface brightness than with infrared color temperatures such as $\nu I_{\nu}(70) / \nu I_{\nu}(160)$ (but see Section 4). Using an alternative measure of infrared surface brightness, $\nu I_{\nu}(70)$, results in a similar declining trend (not shown) with nearly identical dispersion. To test for any dependence on physical scale of the extraction region, we partitioned the region set into two subsamples-those with physical scales above and below $0.65 \mathrm{kpc}$ (the median region size among the sample galaxies). The resulting median trend line of $[\mathrm{C} \mathrm{II}] / \mathrm{TIR}$ versus $\nu I_{\nu}(24)$ remains unchanged between these subsamples, indicating little dependency on physical extraction scale.

At each bin of $\nu I_{\nu}(24)$ above $10^{-7} \mathrm{~W} / \mathrm{m}^{2} \mathrm{sr}$, the standard deviation of regions with that surface brightness is approximately $\sigma([\mathrm{C} \mathrm{II}] / \mathrm{TIR}) \simeq 0.13 \%$. Given that $[\mathrm{C} \mathrm{II}] /$ TIR drops to $\sim 0.2 \%$, this scatter is not insignificant. Rather than being random scatter, however, a single physical parameter appears to be associated with deviation from the fiducial trend: metal abundance.

\subsection{Metallicity}

Even mild changes in metallicity can have strong effects on radiative coupling and dust properties in the ISM of galaxies. As metallicity is reduced, the dust-to-gas ratio drops, at first linearly with metal abundance over a wide range of metallicities (Draine et al. 2007), and then even faster, with growing evidence for nonlinear suppression of dust abundance at metallicities below $12+\log (\mathrm{O} / \mathrm{H}) \sim 8.0$ (Fisher et al. 2014; Rémy-Ruyer et al. 2014), or about one-fifth the solar abundance. $^{22}$ Perhaps not coincidentally, around this same metallicity, the fractional abundance relative to the bulk dust mass of PAH and other small grains (the principal source of photoelectrons which heat neutral gas) drops significantly (Engelbracht et al. 2005; Wu et al. 2006; Draine et al. 2007; Smith et al. 2007; Engelbracht et al. 2008). A reduced abundance of small grains would presumably decrease photoelectric coupling and gas heating, and lower [C II]/TIR.

On the other hand, in galaxies with lower metallicity, stars of a given mass tend to be hotter, leading to increased stellar FUV emission. In addition, the reduced dust opacity may permit a higher fraction of UV starlight, as well as Ly $\alpha$ and other UV lines radiated by $\mathrm{H}$ II regions, to penetrate deep into the neutral gas. These effects could increase the fraction of the energy

\footnotetext{
${ }^{22}$ Assuming $12+\log (\mathrm{O} / \mathrm{H})_{\odot}=8.69$ (Asplund et al. 2009).
} 


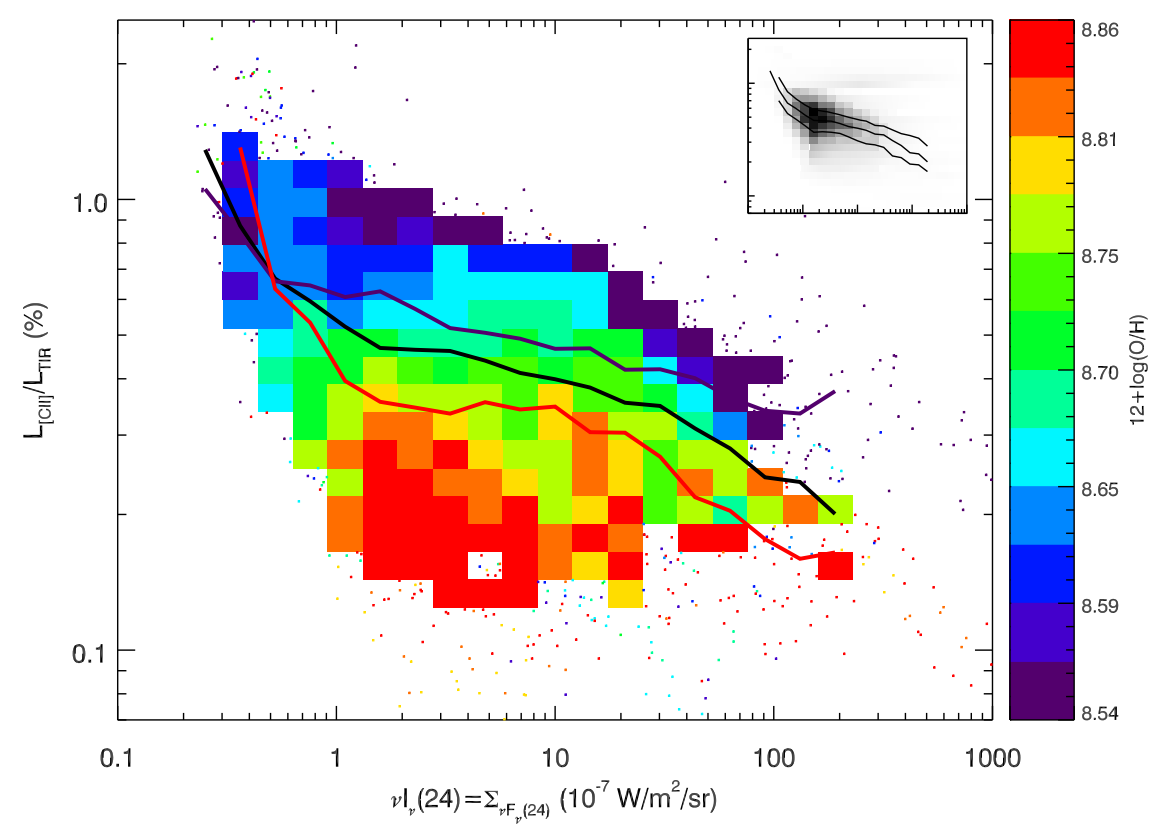

Figure 2. [C II] deficit in approximately 15,000 resolved regions within KINGFISH galaxies vs. the $24 \mu \mathrm{m}$ surface brightness $\nu I_{\nu}(24 \mu \mathrm{m})$. The central $750 \mathrm{pc}$ of AGN host galaxies are excluded. Binning in $\nu I_{\nu}(24)$ and $L_{\mathrm{IC} \mathrm{III}} / L_{\mathrm{TIR}}$ is adopted when nine or more regions lie in a single logarithmic bin. A declining trend with surface brightness is evident, and is independent of the physical scale of the extraction. Colors indicate the binned mean or individually estimated oxygen abundance, $12+$ log $(\mathrm{O} / \mathrm{H})$, from the scale-bar at right. The solid lines indicate the trend lines of median fractional luminosity $L_{\mathrm{IC}}$ II $/ L_{\mathrm{TIR}}$ at each position in binned surface brightness $\nu I_{\nu}(24 \mu \mathrm{m})$. Plotted are the overall median (black), as well as the median of those regions in the top and bottom $10 \%$ of the $12+\log (\mathrm{O} / \mathrm{H})$ range $(\mathrm{color}-\mathrm{indexed}$ to the same abundance scale; $<10$ percentile: dark violet, $>90$ percentile: red). Abundance dispersion within the bins contributes to the offset between these decile trend lines and the locus of bins with matching abundance. In the inset (top right), the linearly scaled density of regions per bin (418 regions maximum) is shown over the same plotting range, together with the median and the inner two quartile lines computed at each bin of surface brightness.

absorbed by dust that is converted to photoelectric heating of the gas, and therefore increase the $[\mathrm{C} \mathrm{II}] /$ TIR ratio.

\subsubsection{The Impact of Metal Abundance on the Deficit}

To investigate the response of $[\mathrm{C} \mathrm{II}] / \mathrm{TIR}$ to changes in abundance, we adopt the $12+\log (\mathrm{O} / \mathrm{H})$ abundance results of Moustakas et al. (2010), who compiled a large number of strong-line oxygen abundance measurements, and present uniformly derived radial gradient and characteristic abundances for the SINGS sample (the Spitzer-based parent sample of KINGFISH, Kennicutt et al. 2003a). Following Moustakas et al., we use the fitted radial abundance gradients, where available, to compute $12+\log (\mathrm{O} / \mathrm{H})$ in each of our regions, and then average the two values obtained using each of the two strong line calibrations the authors chose (a procedure that, for the abundance range in the sample, recovers abundances similar to those measured directly using temperature-sensitive auroral lines; Croxall et al. 2013). For the four galaxies studied not available in the Moustakas et al. sample, we adopted direct or comparable strong-line abundance values. ${ }^{23}$ Characteristic galaxy abundances ranged from 0.17-2.85 times the solar value, and the regions themselves ranged from $12+\log (\mathrm{O} / \mathrm{H})=8.46-8.88(10 \%-90 \%$ range $)$.

Figure 2 demonstrates the impact metallicity has on the fractional luminosity of [C II]. The bins (each containing nine or more distinct regions within the sample) are colored according to the linear mean of their abundance,

\footnotetext{
${ }^{23}$ IC0342: McCall et al. (1985); NGC 3077: Storchi-Bergmann et al. (1994); NGC 2146: K. Croxall (2016, private communication); NGC 5457: Kennicutt et al. (2003b). Note that Moustakas et al. adopt a luminosity-metallicity calibrated abundance for galaxies without adequate line detection, affecting $<8 \%$ of all regions in our sample.
}

$12+\log (\langle\mathrm{O} / \mathrm{H})$. Since gas heating and therefore $[\mathrm{C} \mathrm{II}]$ cooling depends critically on the coupling of UV/optical photons to small dust grains, and since both the grain-ionizing starlight and the abundance of small grains themselves are affected by varying gas metal content, it is perhaps not surprising that, at a given starlight surface density, the substantial scatter in the [C II] deficit is driven primarily by changes in metallicity.

Although the overall trend is toward deeper deficits with increasing surface brightness, at each surface brightness, regions with lower metallicity typically exhibit increased $[\mathrm{C} \mathrm{II}] / \mathrm{TIR}$, and those with higher metallicities fall to deeper deficits. The modest range of $12+\log (\mathrm{O} / \mathrm{H})$ scaling in Figure 2 reflects the limited dynamic range of abundance in the portion of the KINGFISH sample covered by [C II]. At the highmetallicity end, this limit is related to the chemical enrichment history of the universe-local galaxies do not attain much more than twice solar abundance (Pilyugin et al. 2007; Zahid et al. 2014). At the low end, the dominant effect is limited coverage in the far outskirts of galaxies where metallicities are lowest (e.g., Bresolin et al. 2012, though see Section 6 for a discussion of deficit work in lower metallicity samples). Another impact is the abundance scatter within each bin (a median of $\left.\sigma_{\log (\mathrm{O} / \mathrm{H})}=0.13\right)$, which serves to compress the vertical offset between bins of different metallicity. This perbin scatter may stem in part from uncertainties in the measurement and calibration of abundance gradients in the sample, or from breakdowns in the assumption of purely radial abundance variation. This effect also explains the compression toward the median of the high-metallicity (top 10\%) and lowmetallicity (bottom 10\%) trend lines in Figure 2. Yet even with imperfect abundances with values ranging just over a factor of two, the impact on deviations from the overall deficit trend with surface brightness are clear. 


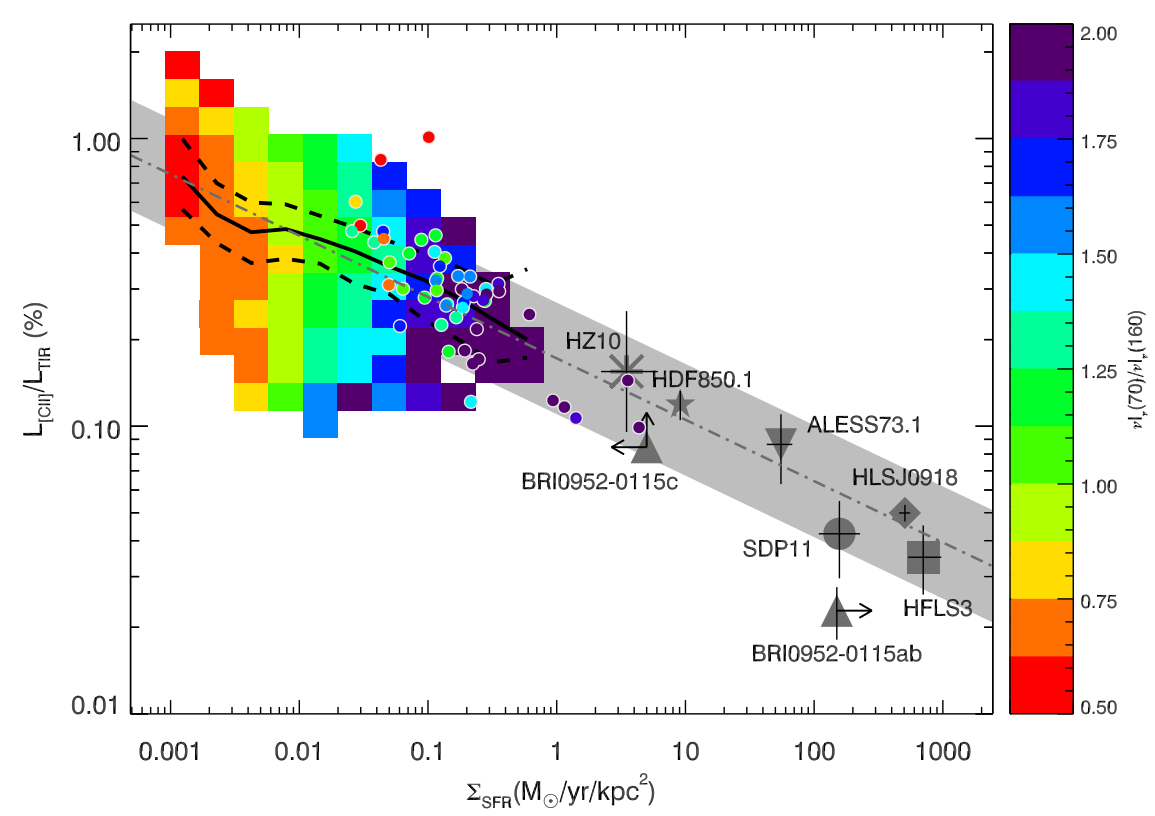

Figure 3. Trend in [C II] deficit with star formation rate density for KINGFISH regions, binned using the methodology of Figure 2. Also included are well-resolved nearby luminous infrared galaxies from the GOALS survey (filled circles), and selected high-redshift sources from $z=1.8-6.4$ with resolved [C II] emission (gray points). The KINGFISH and GOALS sources are color-coded by their binned median or individual dust color temperatures $\nu I_{\nu}(70) / \nu I_{\nu}(160)$, indicated on the color scale at right. The median and upper and lower quartile trend lines are shown for the KINGFISH sample (solid and dashed lines, respectively). These give a better impression of the true scatter of resolved regions about the trend, as the binning method accentuates low-density outlier regions. A fit to the binned median, GOALS, and high-redshift points is shown as the continuous (dot-dashed) line, with the underlying fit uncertainty shaded gray. High-redshift sources from Gallerani et al. (2012) - BRI 0952-0115, $z=4.4$, unlabeled upward triangles; Walter et al. (2012)—HDF850.1, $z=5.2$; Riechers et al. (2013) - HFLS3, $z=6.34$; Bussmann et al. (2013) and Ferkinhoff et al. (2014)—SDP11, $z=1.8$; Rawle et al. (2014)—HLSJ0918(Ra), $z=5.234$; De Breuck et al. (2014)—ALESS73.1, $z=4.8$; and Capak et al. (2015)-HZ10, $z=5.66$.

\subsubsection{Radial Dependence of the Deficit}

Several studies have identified trends of increasing [C II]/ TIR with radius in star-forming galaxies (Madden et al. 1993; Nikola et al. 2001; Kramer et al. 2013; Kapala et al. 2015). Since abundance is assumed to drop monotonically with radius, it is natural to question whether or not $12+\log (\mathrm{O} / \mathrm{H})$ is acting merely as a surrogate for some other physical property of galaxies, which varies radially (aside, of course, from the radial variations in infrared surface brightness itself). Systematic uncertainties in the determination of $12+\log (\mathrm{O} / \mathrm{H})$ gradients are also a potential concern. To investigate these effects and the significance of the deficit dependence on metallicity, we used two approaches. First, we suppressed all radial abundance gradients, and adopted in their place a single characteristic abundance for each sample galaxy, drawn from the stripaveraged values of Moustakas et al. (2010). Even when only a single characteristic abundance value per galaxy is used, the same basic pattern recurs: at a given infrared surface brightness, increasing oxygen abundance drives the deficit to deeper values. We also directly investigated the residuals in [C II]/TIR by subtracting a smoothed spline fit to the median line with $24 \mu \mathrm{m}$ surface brightness in Figure 2 . We found that the residuals from the overall trend with $\nu I_{\nu}(24)$ correlate significantly more strongly with $12+\log (\mathrm{O} / \mathrm{H})$ than with either physical radius or scaled radius (relative to the optical diameter along the major axis).

\section{STAR FORMATION RATE DENSITY ACROSS REDSHIFT}

The areal density with which galaxies form new stars fundamentally impacts their physical character, altering the temperature, pressure and kinetic state of interstellar gas, the size and distribution of molecular clouds, and the efficiency of star formation. In star-forming galaxies, on scales near or above a kiloparsec, $\Sigma_{\text {SFR }}$ varies over a remarkably wide range, from below $10^{-5} M_{\odot} \mathrm{yr}^{-1} \mathrm{kpc}^{-2}$ in nearby low-efficiency dwarf galaxies (e.g., Cook et al. 2012), up to "maximal starbursts" which are Eddington-limited by the impact of radiation pressure on dust at $\Sigma_{\mathrm{SFR}} \simeq 1000 M_{\odot} \mathrm{yr}^{-1} \mathrm{kpc}^{-2}$ (Thompson et al. 2005). Given the strong demonstrated dependency of [C II]/TIR on $24 \mu \mathrm{m}$ surface brightness, it is natural to consider whether or not $\Sigma_{\mathrm{SFR}}$ itself underlies this trend.

Figure 3 explores the dependence of the [C II] deficit on $\Sigma_{\text {SFR. }}$. It combines the resolved regions within KINGFISH galaxies (on physical scales ranging from $0.2-1.6 \mathrm{kpc}$ ) with central values from resolved local LIRGs and ULIRGs from the GOALS sample of Díaz-Santos et al. (2013), as well as a sample of high-redshift galaxies at $z=1.8-6.4$ with directly measured physical size scales from well-resolved [C II] and continuum interferometic measurements. For the KINGFISH galaxies, $\Sigma_{\mathrm{SFR}}$ is obtained from a hybrid indicator, which combines FUV and $24 \mu \mathrm{m}$ surface brightness of Hao et al. (2011). ${ }^{24}$ In the central GOALS regions, $\Sigma_{\mathrm{SFR}}$ is obtained directly from $24 \mu \mathrm{m}$ surface brightness using the prescription of Rieke et al. (2009). In both cases, measurements are performed at a matched common resolution of the PACS $160 \mu \mathrm{m}$ beam (11"'2). Since GOALS sources are typically small compared to the $160 \mu \mathrm{m}$ beam, this required substantially trimming the sample to include only the 51 galaxies with well-resolved Spitzer/MIPS $24 \mu \mathrm{m}$ and Spitzer/IRS $15 \mu \mathrm{m}$ continuum emission (measured along the slit, see Díaz-Santos et al. 2013).

\footnotetext{
${ }^{24}$ Compared to $24 \mu \mathrm{m}$ only calibrations, the addition of FUV photometry increases $\Sigma_{\mathrm{SFR}}$ only below $\sim 0.01 M_{\odot} \mathrm{yr}^{-1} \mathrm{kpc}^{-2}$, and typically by less than $30 \%$.
} 
The resolved KINGFISH regions (colored bins, omitting lowdensity points as well as AGN centers, as in Figure 2) and individual GOALS galaxies are color-coded by their (mean) dust color temperature $\nu I_{\nu}(70 \mu \mathrm{m}) / \nu I_{\nu}(160 \mu \mathrm{m})$. In the case of GOALS, this color temperature is derived from the nearby $63 \mu \mathrm{m}$ and $158 \mu \mathrm{m}$ continuum fluxes underlying the [O I] and [C II] lines, using an average Dale \& Helou (2002) template to account for the slight wavelength offsets. A clear trend of increasing color temperature at higher $\Sigma_{\mathrm{SFR}}$ is evident, although with some mixing between the two-bins of fixed values of $\nu I_{\nu}(70) / \nu I_{\nu}(160)$ ratio do not lie at a single $\Sigma_{\mathrm{SFR}}$, and the scatter of color temperature at a given bin of $\Sigma_{\mathrm{SFR}}$ is significant: $\sigma\left(\nu I_{\nu}(70) / \nu I_{\nu}(160)\right)=0.38$. The median and inner quartile trends of the resolved KINGFISH regions are shown in solid and dashed lines. The higher star formation rate density GOALS sample clearly extends the [C II]/TIR decline set by these resolved regions.

High-redshift sources (see Figure 3 caption) were included directly from reported $\Sigma_{\text {SFR }}$ values or computed from sizes and IR luminosities, typically based on SED-fitting of several IR continuum measurements and utilizing the resolved physical sizes from $[\mathrm{C} \mathrm{II}]$ and nearby continuum maps. Where necessary, conversion to TIR luminosities from FIR (42-122 $\mu \mathrm{m}, 2.4$ times) were made using the M82 template of Polletta et al. (2007), and from IR (8-1000 $\mu \mathrm{m}, 1.06$ times) using the mean template of Dale \& Helou (2002). In all cases expected uncertainties are less than $20 \%$ for conversion among these broad infrared luminosity bands. The TIR luminosity was further converted to star formation rate using the updated scaling of Murphy et al. (2011). Source sizes were computed from the FWHM area of elliptical Gaussian fits to the [C II] and/or resolved rest-frame sub-millimeter continuum intensity maps (averaging the two where both were available).

Taken together with resolved lower surface brightness KINGFISH and GOALS samples, the high-redshift galaxies extend a single trend of deepening [C II] deficit over more than six orders of magnitude in $\Sigma_{\mathrm{SFR}}$. Of particular interest are the two galaxies which form part of the single galaxy group BRI 0952-0115 (upward gray triangles in Figure 3, Gallerani et al. 2012). These two galaxies have approximately the same infrared luminosity of a few times $10^{12} L_{\odot}$, but one is approximately 10 times more extended, and thus, with its correspondingly lower $\Sigma_{\mathrm{SFR}}$, has a much higher limiting [C II]/ TIR ratio.

A single power-law fit to the binned KINGFISH regions, GOALS galaxies, and high-redshift sources is shown as the dot-dashed line in Figure 3. The relatively tight correlation permits inverting the relation to approximately estimate $\Sigma_{\mathrm{SFR}}$ directly from a measured value of the [C II] fractional line luminosity:

$$
\Sigma_{\mathrm{SFR}}=(12.7 \pm 3.2)\left(\frac{L_{[\mathrm{C} \mathrm{II}]} / L_{\mathrm{TIR}}}{0.1 \%}\right)^{-4.7 \pm 0.8}\left(\frac{M_{\odot}}{\mathrm{yr} \mathrm{kpc}^{2}}\right) .
$$

This relationship applies when young stars provide the dominant energy source, and only on scales greater than a few hundred parsecs. The slope is similar whether the fit is performed to local sources only, or with high-redshift sources included. At the highest physically meaningful $\Sigma_{\mathrm{SFR}}$ of $\sim 1000$ $M_{\odot} \mathrm{yr}^{-1} \mathrm{kpc}^{-2},[\mathrm{C}$ II $] / \mathrm{TIR} \simeq 0.03 \%-0.045 \%$. Since the slope is relatively steep, care must be taken to correctly account for input uncertainties in $[\mathrm{C} \mathrm{II}] / \mathrm{TIR}$. Even with minimal input uncertainties, the uncertainty in the relation itself contributes to over an order-of-magnitude range in the resulting $\Sigma_{\mathrm{SFR}}$ values (see gray band in Figure 3 ). The steepness of the $\Sigma_{\mathrm{SFR}}-L_{[\mathrm{C} \text { II] }} /$ $L_{\mathrm{TIR}}$ relation also implies that, in blind [C II] surveys, lower $\Sigma_{\text {SFR }}$ galaxies will be overrepresented compared to more compact star-forming systems.

An important advantage of Equation (1) is that, in addition to applying to regions within resolved galaxies, it can be used with the observed global luminosity ratio $L_{[\mathrm{C} \text { II }]} / L_{\mathrm{TIR}}$ to estimate the star formation surface density $\Sigma_{\mathrm{SFR}}$ if the [C II] and TIR emitting size scales are similar (as they are in resolved local galaxies; de Blok et al. 2016). It is important to note that Equation (1) is not a calibration for total star formation rate in a galaxy, as galaxies with widely differing SFR can have similar $\Sigma_{\text {SFR }}$. If, however, the total star formation rate is known separately, Equation (1) permits estimation of the compactness of a given star-forming system.

\section{THE AGN IMPACT}

AGNs can reduce the relative cooling power of [C II] in galaxies in several ways. They are effective at producing warm central dust continuum emission (up to dust sublimation temperatures of $\sim 10^{3} \mathrm{~K}$ ), which can substantially increase bolometric infrared luminosity and drive an apparent deficit in $[\mathrm{C}$ II $] /$ TIR. They can also directly impact the cooling line emission itself. One direct effect on [C II] emission results from changes in the overall ionization state of the gas. Langer \& Pineda (2015) modeled this effect on [C II] emission in AGNs and found substantially reduced values (to $[\mathrm{C} \mathrm{II}] /$ TIR $\sim 0.01 \%$ ) in the inner $\sim 10^{2}$ pc of AGN hosts. Another effect is the possible photo-destruction of small dust grains by X-rays out to kiloparsec scales (Voit 1992). The loss of smaller grains then reduces the efficiency of photoelectric heating of neutral gas by UV photons (whether those photons are generated by stellar or accretion-driven sources).

While KINGFISH sources were not selected to host luminous AGNs, approximately half of the sample, including 26 of the [C II]-targeted galaxies considered here, have nuclear optical classifications consistent with AGNs or composite sources (either Seyferts or LINERs; Moustakas et al. 2010). Although none are bright Seyfert AGNs (with typical broadband X-ray luminosities up to $10^{44} \mathrm{erg} \mathrm{s}^{-1}$ ), the spatial resolution available in KINGFISH (down to $\sim 200 \mathrm{pc}$ ) means that any AGN impact on the cooling balance can be directly resolved.

Figure 4 illustrates the striking behavior of [C II] in the inner $1.5 \mathrm{kpc}$ of several AGN hosts in the sample. The steep radial trend of increasing deficit toward the centers of these three galaxies is immediately evident. One of these, NGC 4736, is the example map shown in Figure 1, where the strong deficit of [C II] relative to underlying infrared continuum can be readily seen in the central region, dropping in this case to [C II]/ TIR $\sim 0.05 \%$. In Figure 4, the ratio [C II]/TIR is normalized for each galaxy at a de-projected nuclear distance of $1.5 \pm 0.2 \mathrm{kpc}$, and these three galaxies in particular show a similar pattern of rapidly declining $[\mathrm{C} \mathrm{II}] / \mathrm{TIR}$ values below $\sim 1 \mathrm{kpc}$. While the surface brightness $\nu I_{\nu}(24)$ of these AGN hosts does increase toward the center (color intensity scale of the points), the relative decline in $[\mathrm{C} \mathrm{II}] /$ TIR for the modest range in brightness from disk to nucleus is substantially steeper than the general trend shown in Figure 2. To demonstrate this, we have calculated the expected change in the normalized deficit with nuclear distance due purely to changing $24 \mu \mathrm{m}$ surface 


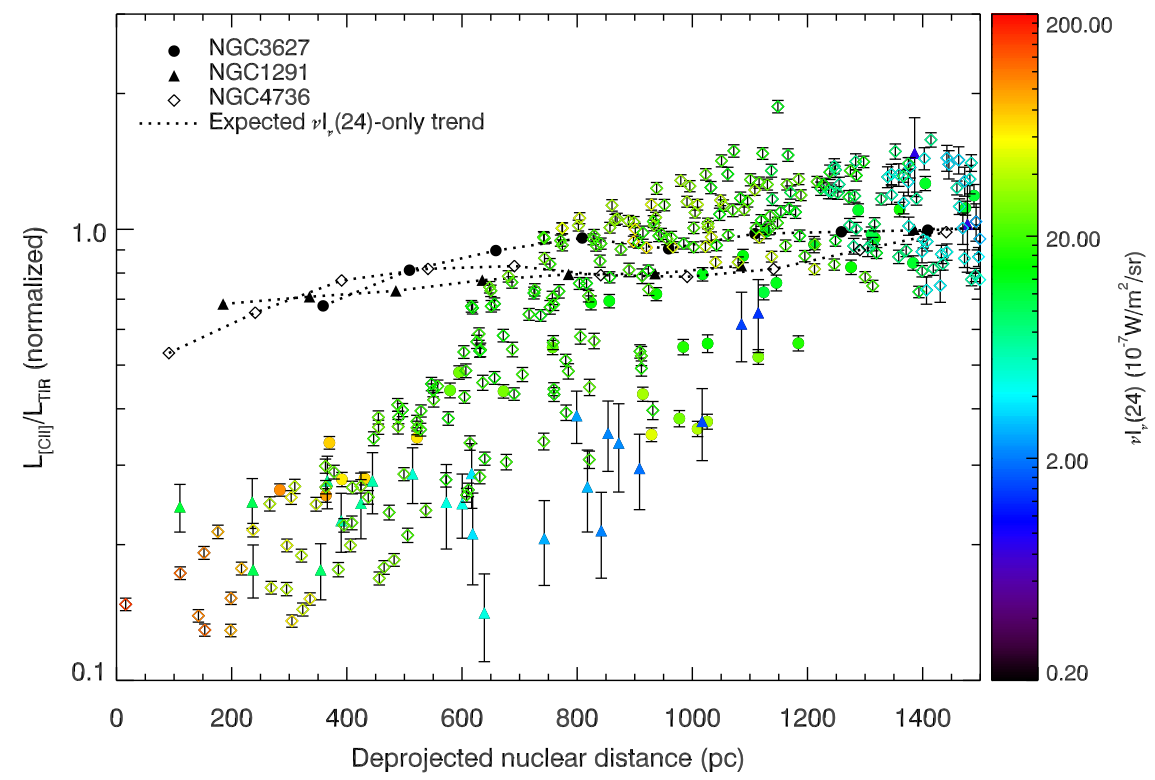

Figure 4. De-projected radial distribution of [C II]/TIR in the inner $1.5 \mathrm{kpc}$ of three AGN systems with strong central [C II] suppression. In each galaxy, [C II]/TIR has been normalized to its value in a $400 \mathrm{pc}$ wide annulus centered at $1.5 \mathrm{kpc}$ distance. Individual regions are color-coded by $24 \mu \mathrm{m}$ surface brightness. The expected modest inward decline of [C II]/TIR (normalized), given the increase in $24 \mu \mathrm{m}$ surface brightness toward the centers of each galaxy is shown by the dotted lines, computed in $150 \mathrm{pc}$ bins using the median $\nu I_{\nu}(24)-[\mathrm{C} \mathrm{II}] / \mathrm{TIR}$ trend line of Figure 2. Points within $750 \mathrm{pc}$ of the centers of these and other AGN hosts were omitted from Figures 2 and 3.

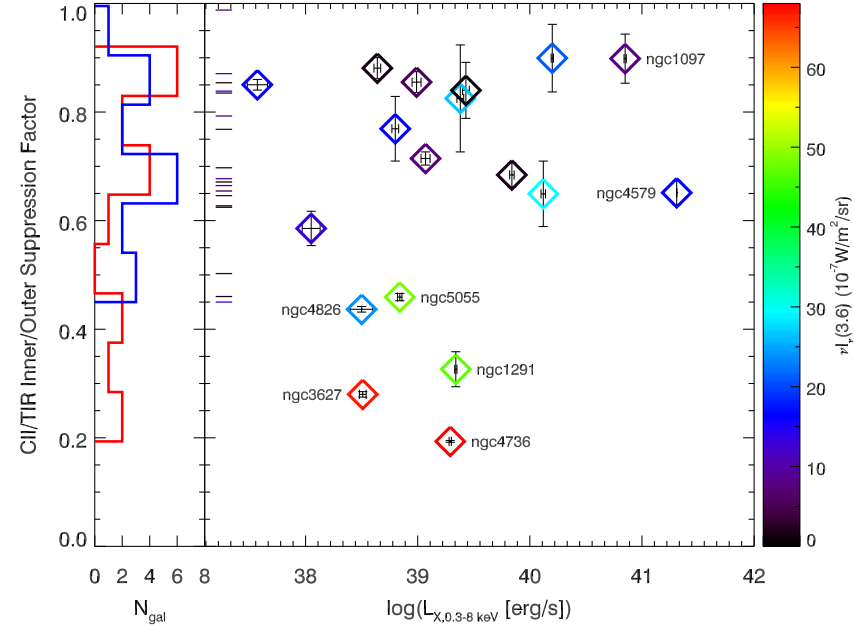

Figure 5. Central suppression factor of [C II] cooling power, defined as the ratio of the inner [C II]/TIR value (averaged within $400 \mathrm{pc}$ of the center) to the outer value (averaged over an annulus at $1-2 \mathrm{kpc}$ ). A suppression factor of 1 indicates no change in $[\mathrm{C} \mathrm{II}] / \mathrm{TIR}$ from outer regions to the center. The marginal histogram on the left shows the distribution of suppression factors in AGN (red) and non-AGN (blue) hosts, and on the right, as a function of central $0.3-8 \mathrm{keV}$ X-ray luminosity. The [C II] central suppression factors in non-AGN host centers are also shown as color-coded bars near the vertical axis. All galaxies are color-coded by central average $\nu I_{\nu}(3.6 \mu \mathrm{m}$ ) (a surrogate for evolved starlight intensity).

brightness. For each galaxy, we apply the median trend line of [C II]/TIR with $\nu I_{\nu}(24)$ (Figure 2) to the average region-byregion surface brightness. We bin radially in nuclear distance bins of $150 \mathrm{pc}$, and normalize [C II]/TIR as before at $1.5 \pm 0.2 \mathrm{kpc}$. These expected trends are shown in Figure 4 as the dotted black lines, which clearly demonstrate how strong the observed central suppression is in these AGN hosts, compared to the modest changes that would arise from centrally increasing surface brightness.
One more potential impact of the presence of an AGN on the heating/cooling balance is entirely indirect: especially at low fractional AGN luminosity, the optical diagnostics used to classify nuclear regions are diluted in the presence of star formation. For this reason, most local low-luminosity AGN galaxies are dominated by older stellar populations in their centers (e.g., Singh et al. 2013). In this interpretation, the AGN is relevant only by association with the high starlight intensity and softer spectrum that characterize their host's old central stellar population.

Langer \& Pineda predicted deviations in [C II]/TIR above $\mathrm{X}$-ray luminosities of $10^{43} \mathrm{erg} \mathrm{s}^{-1}$. To investigate trends with AGN luminosity, we draw on the central $0.3-8 \mathrm{keV}$ luminosities from Chandra, which Grier et al. (2011) compiled for the SINGS sample. Figure 5 shows the central suppression of [C II]/TIR, defined as the ratio of this value in the central $400 \mathrm{pc}^{25}$ relative to its average value at $1-2 \mathrm{kpc}$. It is immediately evident that, while all AGN host galaxies show some suppression of [C II]/TIR toward their centers, in most cases this suppression is modest, approximately $30 \%$. The strength of [C II] suppression does not correlate with central $\mathrm{X}$-ray luminosity, and for most AGNs, it is similar to non-AGN hosts in the sample $-30 \% \pm 15 \%$ (seen as bars at left and in the marginal histogram). But a small number of AGN sources (including the three with radial profiles shown in Figure 4) show substantially higher central suppression factors, with [C II]/TIR reduced by up to a factor of five from disk to center. The five most suppressed sources have $0.3-8 \mathrm{keV}$ luminosities well below the average $\left(L_{0.3-8 \mathrm{keV}}=10^{40.3} \mathrm{erg} \mathrm{s}^{-1}\right)$. Moreover, these highly suppressed sources are in fact those with the largest central surface densities of evolved starlight, as measured by $\nu I_{\nu}(3.6 \mu \mathrm{m})$ (color scale of the points). NGC 4736, the low-luminosity AGN host with the strongest central $[\mathrm{C} \mathrm{II}] /$ TIR suppression, has the highest central $3.6 \mu \mathrm{m}$

\footnotetext{
${ }^{25}$ Or, the central pointing for the few galaxies with beam sizes larger than $400 \mathrm{pc}$.
} 
surface brightness of the entire sample. This suggests that the high density of soft starlight may drive the enhanced central [C II]/TIR suppressions in AGN hosts.

\section{DISCUSSION}

Relative to integrated infrared luminosity, the fractional power emitted in [C II] - the dominant neutral gas coolant and most luminous emission line in most galaxies-ranges over more than two orders of magnitude, from [C II]/TIR $\gtrsim 1 \%$ to $\lesssim 0.01 \%$. This "cooling line deficit" has been well studied globally in galaxies ranging from normal star-forming to ultraluminous systems, with the physical origin as yet unresolved. Such global deficits have been invoked as evidence in support of distinct physical conditions controlling the star formation process in compact luminous galaxies, versus "mainsequence" galaxies forming stars at low surface densities.

A number of physical explanations for the large variations in the power of the dominant cooling line relative to dustreprocessed emission among galaxies have been considered, including the following.

1. [C II] self-absorption, which could lead to absorbed velocity-resolved line profiles in deep deficit sightlines.

2. Strong continuum extinction at $158 \mu \mathrm{m}$.

3. Significant and varying contributions of [C II] from (hydrogen-) ionized gas (e.g., Luhman et al. 2003).

4. Collisional quenching of [C II] emission at high density, which would result in the dense gas coolant [O I] compensating (e.g., Brauher et al. 2008), although this effect was not seen in two KINGFISH galaxies (Croxall et al. 2012).

5. H II regions with high ionization parameter, in which dust absorbs non-ionizing UV photons before they can enter neutral gas. This should result in deficits in all cooling lines for neutral gas (see Graciá-Carpio et al. 2011).

6. Small grain destruction or charging (e.g., Malhotra et al. 2001), which reduces photoelectric yield and should result in correlations between [C II]/TIR or the [C II]/PAH ratio and, e.g., $q_{\mathrm{PAH}}$ (the fractional mass in PAH grains, Draine \& Li 2007), or PAH band ratios indicative of ionization such as $7.7 \mu \mathrm{m} / 11.3 \mu \mathrm{m}$ (as found by Croxall et al. 2012).

7. The collisional coupling of gas and dust grains at high density, which can "short-circuit" the heating/cooling balance via continuum cooling at high gas densities (Spaans \& Silk 2000).

8. AGN impacts on the ionization state of gas (see Section 5, and Langer \& Pineda 2015), which would lead to a correlation between deficits and central X-ray luminosity.

9. An infrared excess relative to the rate radiation couples to the gas, for example in energetically dominant AGN/ quasars, at extreme dust column densities, or in the regions of high starlight intensities that are dominated by softer spectra from old stellar populations in central galaxy bulges (e.g., Groves et al. 2012; Draine et al. 2014).

10. Other cooling channels such as rotational $\mathrm{CO}$ emission dominating the gas-phase cooling power, for example at low ratios of UV field to gas density, where the atomic gas layer becomes thin and the [C II] line intensity diminishes (see Wolfire et al. 1989; Kaufman et al. 1999).
CO does not, however, appear to provide substantial global cooling in ULIRGs (Rosenberg et al. 2015).

Whatever combination of the above physical effects drives the deficit, here we have demonstrated that the same varying deficit of cooling power operates within galaxies on scales as small as $200 \mathrm{pc}$. Remarkably, a single physical property controls the bulk of this variation over six orders of magnitude: the star formation rate density, with increasing $\Sigma_{\mathrm{SFR}}$ driving down the fractional [C II] cooling power.

Due to its high intrinsic luminosity, and accessibility at high redshift (e.g., Carilli \& Walter 2013), there is considerable interest in employing [C II] emission as a direct tracer of star formation. The strong deficit dependence on $\Sigma_{\mathrm{SFR}}$ has ramifications on the use of [C II] as a star formation indicator. Resolved studies that explore $\Sigma_{\mathrm{SFR}}-I_{[\mathrm{C} \text { II] }}$ surface brightness relationships effectively "calibrate out" this dependence and as a result can yield relatively unbiased indicators (e.g., HerreraCamus et al. 2015, who found that the [C II]-SFR relation is better behaved in surface density than luminosity space). Applying these resolved [C II]-SFR calibrations to global luminosities is more uncertain, unless the sample exhibits a modest range of global $\Sigma_{\mathrm{SFR}}$ similar to the galaxies used for the calibration. This fact is easily understood when considering the extreme range of [C II]/FIR found at fixed FIR luminosity $(\gtrsim 250 \times$, Brisbin et al. 2015). The strong trend seen in Figure 2 also explains the residual dependence of global [C II]/SFR on surface brightness and dust color temperatures (e.g., De Looze et al. 2014; Herrera-Camus et al. 2015). Even the definition of $\Sigma_{\text {SFR }}$ itself requires some care, as different observational size scales for extended star-forming disks can yield appreciable differences. The results here apply on size scales above $200 \mathrm{pc}$ and within the main [C II]-emitting disk of galaxies.

At a given surface brightness, deficit variations are found that are not random but are correlated with the gas-phase oxygen abundance, with decreasing metallicity associated with increasing $[\mathrm{C} \mathrm{II}] / \mathrm{TIR}$. These variations are consistent with the large measured fractional luminosities of [C II] in some very low-metallicity objects (e.g., Hunter et al. 2001; Cormier et al. 2010; Cigan et al. 2016). In contrast, Cormier et al. (2015) found little dependence of $[\mathrm{C} \mathrm{II}] /$ TIR on $12+\log (\mathrm{O} / \mathrm{H})$ in global measurements of a large sample of low-metallicity dwarf galaxies. Since the $\Sigma_{\mathrm{SFR}}$ dependence is stronger than the impact of metallicity, unresolved global measures sampling widely varying local radiation field strengths might wash out these residual trends. Vallini et al. (2015) modeled [C II] emission at high redshift, and found a second-order dependence of the predicted [C II]-SFR relation with metallicity $Z$, which has the opposite sense of our observations (at a fixed SFR, $L_{[\mathrm{C} \text { II] }}$ increases in their models as metallicity increases), but this offset may arise principally from the adopted correlation between baryonic overdensity and $Z$.

The apparent cooling line excess with decreasing metallicity at a given surface brightness (Figure 2) is difficult to reconcile with the plummeting relative abundance of small dust grains at low metallicity. Since PAHs and related small grains provide the bulk of photoelectric heating, finding a cooling line excess relative to large grain heating near the metallicity $12+\log (\mathrm{O} / \mathrm{H}) \simeq 8.2$ where PAHs appear to begin disappearing (Engelbracht et al. 2005; Wu et al. 2006; Draine et al. 2007; Smith et al. 2007; Engelbracht et al. 2008) indicates that some other process must contribute. One possibility is that the contribution of [C II] from ionized gas simultaneously increases 
as metallicity drops. This has been investigated in detail by K. Croxall et al. (2017, in preparation) using [N II] $205 \mu \mathrm{m}$ emission (which shares [C II]'(s) critical density in ionized gas and thus tracks it closely). They find that only a modest fraction $(<\sim 40 \%)$ of $[\mathrm{C} \mathrm{II}]$ originates in ionized gas and that this fraction in fact decreases significantly as metallicity drops, essentially compounding the problem. It may also be the case that other small grains with abundances that are insensitive to declining metallicity and for which PAH grains do not act as observational surrogates can still contribute substantial heating (e.g., Galliano et al. 2005; Israel \& Maloney 2011). Cosmic rays may also play a role, if their heating contribution (relative to dust photoelectric heating) increases at low metallicity. This could occur, for example, due to (1) an increasing fraction of energy going into cosmic rays as a result of reduced radiative cooling in supernova blastwaves, and (2) reduced absorption of FUV photons (and consequent photoelectric heating) in lowmetallicity galaxies with lower dust abundances. A final explanation is that [C II] emission at low metallicity is dominated by larger "[C II]-regions" surrounding hydrogen ionization zones, made possible by the increased penetration depth of photons with $h \nu \lesssim 13.6 \mathrm{eV}$ in the reduced dust environments, which can maintain a larger fraction of the ISM's carbon as $\mathrm{C}^{+}$, and/or increase the grain heating efficiency due to a more dilute radiation field heating the gas (e.g., Poglitsch et al. 1995; Madden et al. 1997; Israel \& Maloney 2011). A similar argument based purely on scaling radiation density and resulting cloud structure is given by Narayanan \& Krumholz (2016). In strong recombination regions, the abundance of $\mathrm{C}^{+}$could also be enhanced by the reduced rate of carbon recombination on grain surfaces expected as the small grain abundance drops (Kaufman et al. 1999). Further discriminating among the physical mechanisms underlying the deficit's response to metallicity may require partitioning [C II] emission into its ionized, neutral, dense, and diffuse emitting environments.

Some studies have suggested that the [C II] deficit arises solely from the impact of AGNs on the cooling balance in the ISM (e.g., Sargsyan et al. 2012, but see Ibar et al. 2015 for an opposing view). The continuity of the presented deficit trend from hyperluminous high-redshift galaxies down to subkiloparsec scales within normal star-forming galaxies demonstrates this not to be the case generally. While there is a strong central deficit impact of some AGNs in the KINGFISH sample, as discussed in Section 5, it is likely indirect, related to the high density of low-energy starlight photons, which accompany lower luminosity AGNs. As modeled by Draine et al. (2014) for M31's bulge, the softening central starlight spectrum couples less efficiently to the PAHs than to the bulk grain populations. This presumably would lead to reduced photoelectric heating efficiency of the gas, as less photo-ionization energy per photon is available when the average photon energy drops. A detailed model of this effect with varying starlight spectra could provide a valuable test of this scenario.

At low luminosity, a related issue could be the recently revealed ambiguous power source of many LINER galaxies (with resolved studies showing extended LINER-like emission inconsistent with black hole accretion, Singh et al. 2013; Belfiore et al. 2016). The optical classification scheme of Moustakas et al. (2010) we utilize did not separate Seyfert and LINER types. Whether other expected direct impacts of AGNs, for example on the kiloparsec-scale carbon ionization state or the widescale small grain population, can be disentangled from the effects of changing starlight conditions in the centers of AGN hosts is unknown, but resolved [C II] investigations in AGNs with X-ray luminosities above $10^{43} \mathrm{erg} \mathrm{s}^{-1}$ would be illuminating.

At the highest luminosities, the dust continuum of [C II]emitting galaxies can be dominated by powerful AGNs, rendering Equation (1) inapplicable. An example of this is a galaxy at $z=4.6$ with the highest currently measured infrared luminosity $\left(L_{\mathrm{TIR}} \sim 2.2 \times 10^{14} L_{\odot}\right.$, Díaz-Santos et al. 2016). Like other high-luminosity systems that host extreme AGNs, it has a resolved continuum size much smaller than its [C II] disk, and a remarkably deep [C II] deficit ([C II]/TIR $\sim 0.003 \%$ ). For such a deep deficit, Equation (1) would imply an unphysical star formation rate density in excess of $10^{7} M_{\odot} \mathrm{yr}^{-1} \mathrm{kpc}^{-2}$. Even using a star-forming SED model to compute a reduced $L_{\mathrm{TIR}}$ by scaling from the much lower FIR (42-122 $\mu \mathrm{m}$ ) luminosity, which is often argued to suffer less contamination from AGN-heated dust than the bolometric infrared, leads to $\Sigma_{\mathrm{SFR}} \gtrsim 10^{5} M_{\odot} \mathrm{yr}^{-1} \mathrm{kpc}^{-2}$. Very deep deficits and the resulting unrealistically high $\Sigma_{\mathrm{SFR}} \mathrm{S}$ implied by Equation (1) likely indicate the presence of dominant AGN heating.

\section{CONCLUSIONS}

We have explored [C II] emission in approximately 15,000 regions within 54 KINGFISH galaxies, supplementing these measurements with well-resolved [C II] observations of nearby luminous infrared galaxies and interferometrically resolved high-redshift [C II] emitters. The uncovered physical trends in the deficit connect environments ranging from low surface brightness regions in moderate star-forming systems to extreme starbursts at the theoretical limits of star formation surface density. We find the following.

1. The [C II] deficit, in which [C II]/TIR exhibits large variations contrary to the simple expectations of heating/ cooling balance, is present within galaxies down to scales of $200 \mathrm{pc}$. This strongly implies that whatever underlying process(es) drive the deficit, they are local physical processes of interstellar gas not related to global galaxy properties like bulk luminosity or presence on the galaxy main-sequence.

2. Within normal star-forming galaxies, [C II]/TIR declines markedly from over $1 \%$ down to $\sim 0.1 \%$ as surface brightness $\nu I_{\nu}(24 \mu \mathrm{m})$ increases, with an average value $\langle[\mathrm{C}$ II $] / \mathrm{TIR}\rangle=0.48 \pm 0.21 \%$.

3. At fixed surface brightness, the gas-phase metallicity within galaxies, $12+\log (\mathrm{O} / \mathrm{H})$, correlates with the residual variation in $[\mathrm{C} \mathrm{II}] / \mathrm{TIR}$, with reduced metal abundance associated with higher [C II]/TIR values (i.e., reducing the deficit), and increased metallicity associated with deeper deficits. Given the apparent declining abundance with falling metallicity of the small grains thought to dominate photoelectric heating of the gas, this is a surprising result.

4. The variation of the deficit with $24 \mu \mathrm{m}$ and $70 \mu \mathrm{m}$ surface brightness can be most directly interpreted as a trend with $\Sigma_{\text {SFR }}$. When combining resolved [C II] measurements of luminous infrared and high-redshift galaxies from $z=1.8-6.4$, the trend found in nearby galaxies smoothly extends over more than six orders of magnitude down to 
the maximal starburst at $\Sigma_{\mathrm{SFR}} \sim 1000 M_{\odot} \mathrm{yr}^{-1} \mathrm{kpc}^{-2}$ and $[\mathrm{C} \mathrm{II}] / \mathrm{TIR} \sim 0.03 \%$.

5. By fitting and inverting the relation between [C II]/TIR and $\Sigma_{\mathrm{SFR}}$, the approximate star formation surface density can be estimated using resolved or unresolved measurements of the fractional [C II] luminosity, with the deepest [C II] deficits corresponding to the highest densities of star formation.

6. Unexpectedly large deficits in the resolved KINGFISH sample occur in the centers of several galaxies hosting low-luminosity AGNs, with a very steep radial suppression of [C II]/TIR inwards from de-projected distances of $1.5 \mathrm{kpc}$ to the center of up to a factor of five.

7. While all sample galaxies, including AGN hosts, exhibit deeper deficits in their centers, the typical suppression of [C II]/TIR is modest: $~ 30 \%$ below the $1.5 \mathrm{kpc}$ inner disk average. Those AGN host galaxies with substantially greater central depressions do not host the most luminous AGNs (in terms of $0.3-8 \mathrm{keV}$ X-ray luminosity), but instead harbor central bulges with the highest surface brightness of starlight at $3.6 \mu \mathrm{m}$. This can be explained if the high intensity but softer starlight from the old stellar populations heating the dust in the centers of many AGN hosts drives continuum emission with reduced radiative coupling to the gas.

8. Galaxies with deficits considerably deeper than [C II]/ TIR $\sim 0.01 \%$ imply unphysical star formation rate densities well above several thousand $M_{\odot} \mathrm{yr}^{-1} \mathrm{kpc}^{-2}-$ a potential indicator of dominant AGN contribution to the infrared luminosity.

This work is based in part on observations made with Herschel, a European Space Agency Cornerstone Mission with significant participation by NASA. Support for this work was provided by NASA through an award issued by JPL/Caltech. We thank Steve Hailey-Dunsheath, T. Rawle, and Tanio DiazSantos for advanced access to their compiled [C II] data sets. We also thank them, as well as Gordon Stacey, Carl Ferkinhoff, M. Kapala, and R. Decarli, for helpful discussions which improved this work. J.D.S. gratefully acknowledges visiting support from the Alexander von Humboldt Foundation and the Max Planck Institute für Astronomie as well as support from the Research Corporation for Science Advancement through its Cottrell Scholars program.

\section{REFERENCES}

Aalto, S., Costagliola, F., Muller, S., et al. 2016, A\&A, 590, A73 Aniano, G., Draine, B. T., Calzetti, D., et al. 2012, ApJ, 756, 138 Aniano, G., Draine, B. T., Gordon, K. D., \& Sandstrom, K. 2011, PASP, 123, 1218

Armus, L., Mazzarella, J. M., Evans, A. S., et al. 2009, PASP, 121, 559

Asplund, M., Grevesse, N., Sauval, A. J., \& Scott, P. 2009, ARA\&A, 47, 481 Bakes, E. L. O., \& Tielens, A. G. G. M. 1994, ApJ, 427, 822 Beirão, P., Armus, L., Helou, G., et al. 2012, ApJ, 751, 144 Belfiore, F., Maiolino, R., Maraston, C., et al. 2016, MNRAS, 461, 3111 Boselli, A., Gavazzi, G., Lequeux, J., \& Pierini, D. 2002, A\&A, 385, 454 Brauher, J. R., Dale, D. A., \& Helou, G. 2008, ApJS, 178, 280 Bresolin, F., Kennicutt, R. C., \& Ryan-Weber, E. 2012, ApJ, 750, 122 Brisbin, D., Ferkinhoff, C., Nikola, T., et al. 2015, ApJ, 799, 13 Bussmann, R. S., Pérez-Fournon, I., Amber, S., et al. 2013, ApJ, 779, 25 Capak, P. L., Carilli, C., Jones, G., et al. 2015, Natur, 522, 455 Carilli, C. L., \& Walter, F. 2013, ARA\&A, 51, 105

Cigan, P., Young, L., Cormier, D., et al. 2016, AJ, 151, 14

Cook, D. O., Seth, A. C., Dale, D. A., et al. 2012, ApJ, 751, 100
Cormier, D., Madden, S. C., Hony, S., et al. 2010, A\&A, 518, L57 Cormier, D., Madden, S. C., Lebouteiller, V., et al. 2015, A\&A, 578, A53 Crocker, A. F., Calzetti, D., Thilker, D. A., et al. 2013, ApJ, 762, 79 Croxall, K. V., Smith, J. D., Brandl, B. R., et al. 2013, ApJ, 777, 96 Croxall, K. V., Smith, J. D., Wolfire, M. G., et al. 2012, ApJ, 747, 81 Dale, D. A., \& Helou, G. 2002, ApJ, 576, 159

de Blok, W. J. G., Walter, F., Smith, J.-D. T., et al. 2016, arXiv:1604.08777 De Breuck, C., Williams, R. J., Swinbank, M., et al. 2014, A\&A, 565, A59 De Looze, I., Cormier, D., Lebouteiller, V., et al. 2014, A\&A, 568, A62 Díaz-Santos, T., Armus, L., Charmandaris, V., et al. 2013, ApJ, 774, 68 Díaz-Santos, T., Armus, L., Charmandaris, V., et al. 2014, ApJL, 788, L17 Díaz-Santos, T., Assef, R. J., Blain, A. W., et al. 2016, ApJL, 816, L6 Draine, B. T., Aniano, G., Krause, O., et al. 2014, ApJ, 780, 172 Draine, B. T., Dale, D. A., Bendo, G., et al. 2007, ApJ, 663, 866 Draine, B. T., \& Li, A. 2001, ApJ, 551, 807

Draine, B. T., \& Li, A. 2007, ApJ, 657, 810

Engelbracht, C. W., Gordon, K. D., Rieke, G. H., et al. 2005, ApJL, 628, L29 Engelbracht, C. W., Rieke, G. H., Gordon, K. D., et al. 2008, ApJ, 678, 804 Farrah, D., Lebouteiller, V., Spoon, H. W. W., et al. 2013, ApJ, 776, 38 Ferkinhoff, C., Brisbin, D., Parshley, S., et al. 2014, ApJ, 780, 142

Fisher, D. B., Bolatto, A. D., Herrera-Camus, R., et al. 2014, Natur, 505, 186 Galametz, M., Kennicutt, R. C., Calzetti, D., et al. 2013, MNRAS, 431, 1956 Gallerani, S., Neri, R., Maiolino, R., et al. 2012, A\&A, 543, A114

Galliano, F., Madden, S. C., Jones, A. P., Wilson, C. D., \& Bernard, J.-P. 2005, A\&A, 434, 867

Graciá-Carpio, J., Sturm, E., Hailey-Dunsheath, S., et al. 2011, ApJL, 728, L7 Grier, C. J., Mathur, S., Ghosh, H., \& Ferrarese, L. 2011, ApJ, 731, 60 Groves, B., Krause, O., Sandstrom, K., et al. 2012, MNRAS, 426, 892 Hao, C.-N., Kennicutt, R. C., Johnson, B. D., et al. 2011, ApJ, 741, 124 Helou, G., Malhotra, S., Hollenbach, D. J., Dale, D. A., \& Contursi, A. 2001, ApJL, 548, L73

Herrera-Camus, R., Bolatto, A. D., Wolfire, M. G., et al. 2015, ApJ, 800, 1 Hunter, D. A., Kaufman, M., Hollenbach, D. J., et al. 2001, ApJ, 553, 121 Ibar, E., Lara-López, M. A., Herrera-Camus, R., et al. 2015, MNRAS, 449, 2498

Israel, F. P., \& Maloney, P. R. 2011, A\&A, 531, A19

Kapala, M. J., Sandstrom, K., Groves, B., et al. 2015, ApJ, 798, 24

Kaufman, M. J., Wolfire, M. G., Hollenbach, D. J., \& Luhman, M. L. 1999, ApJ, 527, 795

Kennicutt, R. C., Calzetti, D., Aniano, G., et al. 2011, PASP, 123, 1347

Kennicutt, R. C., Jr., Armus, L., Bendo, G., et al. 2003a, PASP, 115, 928

Kennicutt, R. C., Jr., Bresolin, F., \& Garnett, D. R. 2003b, ApJ, 591, 801

Kramer, C., Abreu-Vicente, J., García-Burillo, S., et al. 2013, A\&A, 553, A114

Krumholz, M. R., Leroy, A. K., \& McKee, C. F. 2011, ApJ, 731, 25

Langer, W. D., \& Pineda, J. L. 2015, A\&A, 580, A5

Luhman, M. L., Satyapal, S., Fischer, J., et al. 2003, ApJ, 594, 758

Lutz, D., Berta, S., Contursi, A., et al. 2015, arXiv:1511.02075

Madden, S. C., Geis, N., Genzel, R., et al. 1993, ApJ, 407, 579

Madden, S. C., Poglitsch, A., Geis, N., Stacey, G. J., \& Townes, C. H. 1997, ApJ, 483, 200

Magdis, G. E., Rigopoulou, D., Hopwood, R., et al. 2014, ApJ, 796, 63

Maiolino, R., Carniani, S., Fontana, A., et al. 2015, MNRAS, 452, 54

Malhotra, S., Helou, G., Stacey, G., et al. 1997, ApJL, 491, L27

Malhotra, S., Kaufman, M. J., Hollenbach, D., et al. 2001, ApJ, 561, 766

McCall, M. L., Rybski, P. M., \& Shields, G. A. 1985, ApJS, 57, 1

Menéndez-Delmestre, K., Blain, A. W., Smail, I., et al. 2009, ApJ, 699, 667

Moustakas, J., Kennicutt, R. C., Jr., Tremonti, C. A., et al. 2010, ApJS, 190, 233

Murphy, E. J., Condon, J. J., Schinnerer, E., et al. 2011, ApJ, 737, 67

Nakagawa, T., Yui, Y. Y., Doi, Y., et al. 1998, ApJS, 115, 259

Narayanan, D., \& Krumholz, M. 2016, arXiv:1601.05803

Nikola, T., Geis, N., Herrmann, F., et al. 2001, ApJ, 561, 203

Pilyugin, L. S., Thuan, T. X., \& Vílchez, J. M. 2007, MNRAS, 376, 353

Poglitsch, A., Krabbe, A., Madden, S. C., et al. 1995, ApJ, 454, 293

Polletta, M., Tajer, M., Maraschi, L., et al. 2007, ApJ, 663, 81

Pope, A., Chary, R.-R., Alexander, D. M., et al. 2008, ApJ, 675, 1171

Rawle, T. D., Egami, E., Bussmann, R. S., et al. 2014, ApJ, 783, 59 Rémy-Ruyer, A., Madden, S. C., Galliano, F., et al. 2014, A\&A, 563, A31 Riechers, D. A., Bradford, C. M., Clements, D. L., et al. 2013, Natur, 496, 329 Rieke, G. H., Alonso-Herrero, A., Weiner, B. J., et al. 2009, ApJ, 692, 556 Rigopoulou, D., Hopwood, R., Magdis, G. E., et al. 2014, ApJL, 781, L15 Rosenberg, M. J. F., van der Werf, P. P., Aalto, S., et al. 2015, ApJ, 801, 72 Roussel, H., Helou, G., Smith, J. D., et al. 2006, ApJ, 646, 841 Sanders, D. B., \& Mirabel, I. F. 1996, ARA\&A, 34, 749

Sargsyan, L., Lebouteiller, V., Weedman, D., et al. 2012, ApJ, 755, 171

Singh, R., van de Ven, G., Jahnke, K., et al. 2013, A\&A, 558, A43 
Smith, J. D. T., Draine, B. T., Dale, D. A., et al. 2007, ApJ, 656, 770 Spaans, M., \& Silk, J. 2000, ApJ, 538, 115

Stacey, G. J., Geis, N., Genzel, R., et al. 1991, ApJ, 373, 423

Stacey, G. J., Hailey-Dunsheath, S., Ferkinhoff, C., et al. 2010, ApJ, 724, 957

Storchi-Bergmann, T., Calzetti, D., \& Kinney, A. L. 1994, ApJ, 429, 572

Symeonidis, M., Vaccari, M., Berta, S., et al. 2013, MNRAS, 431, 2317

Thompson, T. A., Quataert, E., \& Murray, N. 2005, ApJ, 630, 167

Vallini, L., Gallerani, S., Ferrara, A., Pallottini, A., \& Yue, B. 2015, ApJ, 813,36

van der Laan, T. P. R., Armus, L., Beirao, P., et al. 2015, A\&A, 575, A83
Venemans, B. P., McMahon, R. G., Walter, F., et al. 2012, ApJL, 751, L25

Voit, G. M. 1992, MNRAS, 258, 841

Walter, F., Brinks, E., de Blok, W. J. G., et al. 2008, AJ, 136, 2563

Walter, F., Decarli, R., Carilli, C., et al. 2012, Natur, 486, 233

Willott, C. J., Carilli, C. L., Wagg, J., \& Wang, R. 2015, ApJ, 807, 180

Witt, A. N., Gordon, K. D., Vijh, U. P., et al. 2006, ApJ, 636, 303

Wolfire, M. G., Hollenbach, D., \& Tielens, A. G. G. M. 1989, ApJ, 344,770

Wu, Y., Charmandaris, V., Hao, L., et al. 2006, ApJ, 639, 157

Zahid, H. J., Dima, G. I., Kudritzki, R.-P., et al. 2014, ApJ, 791, 130 